
\title{
28 Research Square \\ Efficacy of Duloxetine Compared with Opioid for Postoperative Pain Control Following Total Knee Arthroplasty
}

\section{Man Soo Kim}

Seoul St. Mary's Hospital, The Catholic University of Korea

In Jun Koh

Eunpyeong St. Mary's Hospital, The Catholic University of Korea

Keun Young Choi

Seoul St. Mary's Hospital, The Catholic University of Korea

Sung Cheol Yang

Seoul St. Mary's Hospital, The Catholic University of Korea

Yong In ( iy1000@catholic.ac.kr)

Seoul St. Mary's Hospital, The Catholic University of Korea https://orcid.org/0000-0002-5932-3934

\section{Research article}

Keywords: Duloxetine, Selective serotonin and norepinephrine reuptake inhibitor, Opioid, Pain, Control, Total Knee Arthroplasty

Posted Date: June 4th, 2020

DOI: https://doi.org/10.21203/rs.3.rs-29917/v1

License: @ (1) This work is licensed under a Creative Commons Attribution 4.0 International License. Read Full License

Version of Record: A version of this preprint was published at PLOS ONE on July $2 \mathrm{nd}, 2021$. See the published version at https://doi.org/10.1371/journal.pone.0253641. 


\section{Abstract}

\section{Background}

Opioid is known to be effective in pain control after total knee arthroplasty (TKA). However, recently it has become a major concern due to addiction. Duloxetine, the serotonin-norepinephrine reuptake inhibitor, is effective for pain control in osteoarthritis patients. However, no study has compared the efficacy of the two for pain control after TKA. The purpose of this study was to assess the efficacy of duloxetine as an alternative to opioid treatment for postoperative pain management following TKA.

\section{Methods}

Among 857 patients, 260 (30.3\%) of patients received opioid or duloxetine for pain control for 6 weeks when the pain Visual Analogue Scale (VAS) score was greater than 4 out of 10 at the time of discharge. 121 patients in the Opioid group and 118 in the Duloxetine group were followed up for more than one year. Preoperative and postoperative patient reported outcome measures (pain VAS score, Western Ontario and McMaster Universities OA Index (WOMAC) score, and the rate over the minimum clinically significant difference (MCID) using WOMAC score) were compared. The rate of further drug prescription (opioid or duloxetine) after 6 weeks of first prescription, 30-day readmission rate, and side effects were also investigated.

Results

There was no significant difference in pain VAS score, WOMAC Pain and Function score, or the rate over MCID at each time point between before and after surgery (all $p>0.05$ ). Fifteen (11.0\%) patients in the opioid group and six (4.8\%) patients in the duloxetine group were prescribed additional medication after first 6 weeks, showing no significant $(p>0.05)$ difference in proportion. The 30-day readmission rate and the incidence of side effects were also similar (all $p>0.05$ ).

\section{Conclusion}

Duloxetine has a similar effect to opioid on postoperative pain control. Thus, duloxetine can be considered as an alternative to opioid for postoperative pain control following TKA.

Level of evidence:

Therapeutic study Level $₫$.

\section{Background}

Postoperative pain in early days of recovery is severe after Total Knee Arthroplasty (TKA) [1]. Comprehensive pain regimens are generally used for pain control following TKA [2, 3]. Opioids have been used as an important part of multimodal postoperative analgesic regimen [2, 3]. In particular, oral opioids are effective in relieving postoperative pain after discharge and easily prescribed. However, their use has negatively impacted not only patients, but also our society [4]. Recently, the use of excessive and indiscreet opioids has become a major concern $[5,6]$. Continued use of opioid increases the risk of opioid-related side effect, opioid induced 
hyperalgesia, and addiction [2, 7]. As a result, there has been growing interest in effective methods for postoperative pain control while minimizing the use and side effects of opioids [3].

Selective serotonin and norepinephrine reuptake inhibitor (SNRI) can produce analgesic effect on chronic pain of knee OA [8]. In addition, SNRI is associated with increased morphine's anti-nociceptive activity [9]. Duloxetine, a potent SNRI, can inhibit the reuptake of serotonin and norepinephrine that could modulate descending inhibitory pain pathway in the central nervous system (CNS) [10]. Therefore, theoretically duloxetine can reduce postoperative pain after TKA [11, 12]. Duloxetine is known to have a discontinuation syndrome, not an addiction [13]. It has potential to attenuate opioid-induced withdrawal syndrome in opioid dependence status [14]. The role of this antidepressant on efficacy of postoperative pain control has not been investigated in detail or limited to acute postoperative pain $[11,15]$.

Although limited data have suggested the efficacy of duloxetine in treating acute pain after TKA, there is no study yet on the efficacy of duloxetine as a centrally acting agent compared to opioids in postoperative pain control following TKA $[16,17]$. In addition, its effects on pain relief in short term and long term as part of a multimodal perioperative analgesic protocol for TKA remain unknown $[8,11,15]$. Thus, the purpose of this study was to compare the efficacy of duloxetine with that of opioid as part of a multimodal pain protocol including long-term efficacy of pain relief following TKA. We sought to not only determine whether duloxetine could substitute opioid in multimodal pain relief protocol after discharge, but also compare side effects between duloxetine and opioid.

\section{Patients And Methods}

This retrospective study was approved by the Institutional Review Board of our hospital. All patients provided written informed consent. We identified all patients who underwent unilateral primary TKA with posteriorstabilized (PS) prosthesis by two surgeons in a single institution for the treatment of knee OA between January 2016 and June 2018 with a minimum follow-up of one year. Patients were eligible for inclusion in this study if they met the following criteria: the presence of moderate-to-severe pain at discharge (postoperative one week) defined as average pain intensity score over 4 measured on a $0-10$ Visual Analogue scale (VAS) (0, no pain; 10 , the worst possible pain) and requiring around-the-clock WHO step III opioids (strong opioids), no previous treatment with opioids (i.e., opioid naïve) or duloxetine [18]. Two surgeons used the same critical pathway and basic pain management protocol for patients during hospitalization for one week, but differed in the addition of drugs in moderate to severe pain at discharge. One surgeon continued to use opioids while another surgeon prescribed duloxetine for pain control. Patients were excluded if they had a diagnosis of osteonecrosis, inflammatory arthritis, traumatic $\mathrm{OA}$, flexion contracture greater than $20^{\circ}$, a previous infection, or knee surgery on the operated knee.

The number of knees that received primary TKA at our institution during the index period was 1,368 $(1,181$ patients). One hundred eighty-seven patients ( 374 knees) underwent same day or staged bilateral TKAs. A total of 137 patients were excluded based on exclusion criteria. Therefore, initial candidates for analysis were 857 knees (857 patients) for unilateral TKA. Patient with moderate to severe pain was prescribed Oxycodone/Naloxone 10/5.0 mg (Targin 5/2.5 mg twice daily, Mundipharma co., Cambridge, UK) or duloxetine $30 \mathrm{mg}$ (Cymbalta $30 \mathrm{mg}$ per day, Lilly co., Indianapolis, IN, USA) for six weeks at the time of discharge (postoperative one week). Therefore, 597 knees (597 patients) who received TKA without discharge regimen of 
opioid or duloxetine were also excluded. Of 857 patients with TKAs, $260(30.3 \%)$ received opioid or duloxetine for pain control postoperatively. There were 136 patients in the Opioid group and 124 patients in the Duloxetine group. Among patients who were treated with opioid and duloxetine postoperatively, only patients who used opioid or duloxetine for six weeks after surgery were included in the final analysis (Fig. 1).

All operations were performed by two senior authors of this study under general anesthesia using posterior stabilized (PS) implants with tourniquet inflation to $300 \mathrm{mmHg}$. Bone cement was used for fixation of all components. Both surgeons shared critical pathway except additional pain management regimen. Two hours before the surgery, multimodal oral analgesic drugs containing $200 \mathrm{mg}$ celecoxib and $150 \mathrm{mg}$ pregabalin were administered as a preemptive analgesia. Intraarticular suction catheter was placed in the joint before closing the joint capsule. It was removed within two days postoperatively. Neither surgeons performed periarticular injections or nerve blocks after operation. Instead, all patients received intravenous patient-controlled analgesia (PCA) which was programmed to deliver $1 \mathrm{~mL}$ of a $100-\mathrm{mL}$ solution containing $2000 \mu \mathrm{g}$ fentanyl postoperatively. Intravenous PCA was removed at four days after operation. Once patients restarted oral intake, $10 \mathrm{mg}$ oxycodone, $200 \mathrm{mg}$ celecoxib, $37.5 \mathrm{mg}$ tramadol, and $650 \mathrm{mg}$ acetaminophen were administered every 12 hours for seven days during hospitalization. Intramuscular injection of tridol (50 mg) was injected as an acute analgesic when a patient reported severe pain greater than level 6 on VAS of 0 to 10 during hospitalization period. After discharge, patients received $200 \mathrm{mg}$ celecoxib and $650 \mathrm{mg}$ acetaminophen every 12 hours for six weeks after operation. In addition, patient with moderate to severe pain was prescribed Oxycodone/Naloxone or duloxetine for six weeks at the time of discharge. Additional medication of opioid or duloxetine was prescribed if the patient complained for sustained pain and required additional medication despite six weeks of pain medication after discharge. All patients also received $40 \mathrm{mg}$ of low-molecular-weight heparin (Clexane [enoxaparin]; Sanofi Aventis, Paris, France) subcutaneously for thromboprophylaxis against deep venous thrombosis during postoperative one week. All patients were encouraged to perform active exercises according to our protocol of rehabilitation. Gradually increasing range-of-motion (ROM) and quadriceps-strengthening exercises were started immediately after the surgery. Patients began ambulation using a supportive device on the first postoperative day. Patients wore compression stockings for four weeks after the operation. Follow-up visits were scheduled at six weeks, three months, six months, and one year.

The primary outcome variable was self-reported pain severity with ambulation, which was assessed by using 10-point VAS score. Secondary outcome variables included pain at rest, pain at nighttime, and the Western Ontario and McMaster Universities Osteoarthritis Index (WOMAC) score. To assess the importance of clinical improvement, a 20-point improvement of WOMAC score was evaluated at one year after TKA based on the minimum clinically significant difference (MCID)[19]. The rate of further drug prescription (opioid or duloxetine) after six weeks of first prescription was evaluated. Thirty-day readmission rate was also identified with any reason between the two groups. Finally, adverse events were evaluated at six weeks postoperatively based on previous studies regarding the safety of opioid and duloxetine $[6,8,11,16,20]$.

\section{Statistical Methods}

Primary and secondary endpoints in the two groups were compared. The Shapiro-Wilk test was used to determine normality of data. All data are presented as mean and standard deviation. Comparison of continuous variables between the two groups was performed using unpaired Student's t-test. Fisher exact test was used for 
analysis of categorical variables. Propensity score matching and all statistical analyses were performed using SPSS ver. 21.0 program (SPSS Inc., Chicago, IL, USA). A $p$-value $<0.05$ was considered statistically significant.

\section{Results}

Finally, there were 121 patients in the opioid group and 118 patients in the duloxetine group (Fig. 1).

Characteristic of these patients are shown in Table 1. Demographic data showed no significant difference between two groups (all $p>0.05$ ). Both groups had similar postoperative pain at postoperative six weeks, three months, six months, and one year during ambulation, at nighttime, and at rest (all $p>0.05$ ) (Table 2). ROM did not differ between the two groups preoperatively or postoperatively (at six weeks, three months, six months, or one year) (all $p>0.05$ ) (Fig. 2). 
Table 1

Patient demographics and preoperative characteristics*

\begin{tabular}{|c|c|c|c|}
\hline & Opioid $(n=121)$ & Duloxetine $(n=118)$ & $p$-value \\
\hline \multicolumn{4}{|l|}{ Demographic data } \\
\hline Age (years) & $71.3(7.2)$ & $70.0(7.0)$ & 0.181 \\
\hline Gender (female)† & $105(86.8)$ & $98(83.1)$ & 0.472 \\
\hline $\mathrm{BMI}\left(\mathrm{kg} / \mathrm{m}^{2}\right)$ & $26.0(2.8)$ & $25.5(3.4)$ & 0.200 \\
\hline ASA statust & & & 0.101 \\
\hline 1 & $22(18.2)$ & $33(28.0)$ & \\
\hline 2 & $98(81.0)$ & $82(69.5)$ & \\
\hline 3 & $1(0.8)$ & $3(2.5)$ & \\
\hline Tourniquet time (minutes) & $44.8(11.4)$ & $43.3(10.8)$ & 0.311 \\
\hline \multicolumn{4}{|l|}{ Specific comorbidities } \\
\hline Hypertension & $76(62.8)$ & $74(62.7)$ & 0.987 \\
\hline Diabetes & $22(18.2)$ & $21(17.8)$ & 0.938 \\
\hline Cardiac disease & $15(12.4)$ & $21(17.8)$ & 0.243 \\
\hline Cerebrovascular event & $5(4.1)$ & $10(8.5)$ & 0.191 \\
\hline Thyroid disease & $11(9.1)$ & $8(6.8)$ & 0.509 \\
\hline Kidney disease & $5(4.1)$ & $5(4.2)$ & 0.968 \\
\hline Pulmonary disease & $8(6.6)$ & $8(6.7)$ & 0.586 \\
\hline Liver disease & $7(5.8)$ & $2(1.7)$ & 0.097 \\
\hline Depression & $1(0.8)$ & $2(1.7)$ & 0.619 \\
\hline \multicolumn{4}{|c|}{ * Data are presented as mean (standard deviation). } \\
\hline \multicolumn{4}{|c|}{ † Data are presented as number (percentage). } \\
\hline $\mathrm{BMI}=$ body mass index; $\mathrm{AS}$ & $=$ American Socie & of Anesthesiologist & \\
\hline
\end{tabular}


Table 2

Pain VAS on walking, nighttime, and resting*

\begin{tabular}{|c|c|c|c|c|c|c|c|c|c|}
\hline & \multicolumn{3}{|c|}{ Walking } & \multicolumn{3}{|c|}{ Nighttime } & \multicolumn{3}{|l|}{ Resting } \\
\hline & $\begin{array}{l}\text { Opioid } \\
(n= \\
121)\end{array}$ & $\begin{array}{l}\text { Duloxetine } \\
(n=118)\end{array}$ & $\begin{array}{l}p- \\
\text { valuet }\end{array}$ & $\begin{array}{l}\text { Opioid } \\
(n= \\
121)\end{array}$ & $\begin{array}{l}\text { Duloxetine } \\
(n=118)\end{array}$ & $\begin{array}{l}p- \\
\text { valuet }\end{array}$ & $\begin{array}{l}\text { Opioid } \\
(n= \\
121)\end{array}$ & $\begin{array}{l}\text { Duloxetine } \\
(\mathrm{n}=118)\end{array}$ & $\begin{array}{l}p- \\
\text { valuet }\end{array}$ \\
\hline Preop & $\begin{array}{l}7.2 \\
(1.0)\end{array}$ & $7.1(0.8)$ & 0.162 & $\begin{array}{l}5.2 \\
(1.6)\end{array}$ & $5.3(1.6)$ & 0.421 & $\begin{array}{l}3.8 \\
(1.7)\end{array}$ & $3.6(1.6)$ & 0.347 \\
\hline $\begin{array}{l}\text { POD } \\
6 W\end{array}$ & $\begin{array}{l}3.5 \\
(1.6)\end{array}$ & $3.4(1.5)$ & 0.430 & $\begin{array}{l}3.5 \\
(1.8)\end{array}$ & $3.3(1.6)$ & 0.551 & $\begin{array}{l}1.8 \\
(1.3)\end{array}$ & $1.6(1.2)$ & 0.207 \\
\hline $\begin{array}{l}\text { POD } \\
3 M\end{array}$ & $\begin{array}{l}2.8 \\
(1.4)\end{array}$ & $2.7(1.5)$ & 0.720 & $\begin{array}{l}2.3 \\
(1.6)\end{array}$ & $2.3(1.7)$ & 0.955 & $\begin{array}{l}1.3 \\
(1.3)\end{array}$ & $1.3(1.5)$ & 0.866 \\
\hline $\begin{array}{l}\text { POD } \\
6 M\end{array}$ & $\begin{array}{l}2.2 \\
(0.9)\end{array}$ & $2.2(0.8)$ & 0.245 & $\begin{array}{l}1.5 \\
(1.6)\end{array}$ & $1.1(1.4)$ & 0.080 & $\begin{array}{l}1.1 \\
(1.1)\end{array}$ & $0.9(0.9)$ & 0.115 \\
\hline $\begin{array}{l}\text { POD } \\
1 Y\end{array}$ & $\begin{array}{l}1.8 \\
(1.2)\end{array}$ & $1.9(1.1)$ & 0.682 & $\begin{array}{l}1.1 \\
(1.1)\end{array}$ & $0.9(1.0)$ & 0.063 & $\begin{array}{l}0.7 \\
(0.8)\end{array}$ & $0.6(0.7)$ & 0.252 \\
\hline \multicolumn{10}{|c|}{ * Data are presented as mean (standard deviation). } \\
\hline
\end{tabular}

WOMAC pain score did not differ between the two groups either (all $p>0.05$ ). WOMAC Stiffness, Function, and Total score showed no significant difference between the two groups either at each time point $($ all $p>0.05)$ (Fig. 3). A total of 117 (96.7\%) patients in the opioid group and 114 (96.6\%) in the duloxetine group demonstrated a 20-point improvement in WOMAC score from baseline to postoperative one year, showing no significant difference in proportion $(p=0.971)$.

Fifteen (11.0\%) patients in the opioid group and six (4.8\%) patients in the duloxetine group were prescribed additional medication after first six weeks of pain control using opioid or duloxetine $(p=0.073)$ (Fig. 1). The 30day readmission rate was similar between the two groups (duloxetine group vs. opioid group: 3 (2.5\%) vs. 1 $(0.8 \%), p>0.05)$. Two patients in the duloxetine group and one patient in the opioid group were readmitted for pain control. The one patient in the duloxetine group was due to superficial wound infection.

Although side effects were more frequent in the opioid group than in the duloxetine group, there was no difference in the incidence of side effects between the two groups (all $p>0.05$ ) (Table 3 ). Two patients in the opioid group stopped to take the medication due to nausea/vomiting and drowsiness. One patient in the duloxetine group stopped to take the medication due to drowsiness. 
Table 3

Incidences of adverse events*

\begin{tabular}{|llll|}
\hline Adverse events & Opioid $(\mathbf{n}=\mathbf{1 2 1})$ & Duloxetine $(\mathbf{n}=118)$ & $p$-value \\
\hline Nausea/vomiting & $21(17.4)$ & $14(11.9)$ & 0.274 \\
\hline Constipation & $16(13.2)$ & $13(11.0)$ & 0.693 \\
\hline Dizziness & $21(17.4)$ & $19(16.1)$ & 0.863 \\
\hline Drowsiness & $27(22.3)$ & $22(18.6)$ & 0.524 \\
\hline Headache & $23(19.0)$ & $18(15.3)$ & 0.495 \\
\hline Dry mouth & $27(22.3)$ & $25(21.2)$ & 0.876 \\
\hline *Data are presented as number (percentage) of patients. & \\
\hline
\end{tabular}

\section{Discussion}

The most important finding of this study was that administration of duloxetine for six weeks had similar efficacy to that of opioid for pain and functional improvement following TKA. In addition, duloxetine did not cause major side effects when compared to opioid.

Although post-operative pain was generally less than that before surgery, the duration of post-TKA pain generally continues for six weeks after surgery [17]. Andersen et al. have reported that mean pain score is 2.9 at rest and 3.6 when walking at postoperative two weeks [21]. Pain level is slightly increased at one month after TKA [21]. Although various methods of pain control have been developed, most multimodal pain management protocols have been focused on pain reduction during early postoperative period under hospitalization [22]. Therefore, excessive post-discharge pain following TKA could be a big concern [17, 21]. Multimodal outpatient analgesia regimens after discharge usually include opioids [23-25]. Historically, the use of opioids has been limited to acute pain management or diseases related to chronic pain [26]. However, as thresholds for the use of opioids have gradually decreased, overuse, misuse, and dependency of opioids have become major issues in recent years $[5,6]$. These opioid epidemics are emphasizing the importance of an effective way to improve management of pain control after orthopedic procedure without dependency [27]. Hansen et al. have reported the prevalence of chronic opioid users after TKA and demonstrated that $66.5 \%$ of patients continue chronic opioid use during one year postoperatively [28]. Despite the above data, little is known about the effective substitution of opioids for management of pain after TKA. Therefore, there have been a growing demand for methods to reduce or replace the use of opioids after TKA.

In this study, we found that perioperative use of duloxetine for six weeks in patients who had moderate pain level at discharge showed similar pain control to opioid after TKA during postoperative 1, 3, 6, and 12 months. Several positive attempts have provided improved expectations for the use of antidepressants for postoperative pain control [29]. YaDeau et al. [17] and Ho et al. [16] have studied perioperative use of duloxetine following TKA. They showed that duloxetine could reduce opioid consumption without affecting pain level or side effects $[16,17]$. These findings were partly different from our study results. The reason for such difference in results is unclear. However, it seems to be due to difference in research design using different pain control protocols [16, 17]. Duloxetine does not have an analgesic effect during immediate postoperative period of two weeks after 
TKAs [30]. Therefore, evaluating the efficacy of duloxetine with two weeks might not be an obvious result. Unlike those studies described above, we compared results for up to one year after TKA with the use of the two drugs for six weeks after surgery. This is the first study to identify short- and long-term course of two drugs over a period of time after TKA rather than temporary drug use [16, 17]. Our findings of this study suggest that incorporation of duloxetine into current after-discharge multimodal pain management protocol might be an effective alternative for patients who need to use opioids because of sustained post-discharge pain after TKA.

Our findings also support that perioperative use of duloxetine can provide similar functional improvement in patients undergoing TKA compared to opioid. To date, no studies have compared postoperative functional aspects between opioid and duloxetine groups after TKA [16, 17]. Previous studies of two low quality randomized controlled trials have compared antidepressants, not duloxetine, and opioid in patients with neuropathic pain [31, 32]. There were no significant differences in functional scores between the two groups using SF 36 in either study [31, 32]. This result was consistent with our research. There was no functional difference between the two groups after TKA during one year based on measurement of WOMAC function score. Systemic duloxetine has a role in central pain inhibition as a SNRI in the central nervous system [8]. It appears to have perioperative analgesic effect [16]. In addition, as an antidepressant drug, duloxetine could prevent common transient emotional problems during the perioperative period [12]. The combination of pain relief and better emotional state might contribute to better postoperative functional status [12].

Major barriers to opioid use are opioid-related side effects [33]. The most common side effects are constipation, dizziness, nausea, vomiting, and delirium. Constipation is one major side effect of opioids. The use of oxycodone-naloxone can reduce the rate of constipation [33]. Duloxetine is a drug that can act on the central nervous system like opioids. However, it has distinctly different mechanisms of action and other side effects [34]. The most common side effects of duloxetine are nausea, vomiting, constipation, and dry mouth [35, 36]. In the present study, side effects of duloxetine and opioids were found to be comparable with each other. The frequency of these side effects in the present study was similar to those reported in previous studies [8, 20,37]. Nonetheless, the safety of duloxetine is inconclusive with supporting evidence in the present study. This needs further research.

This study has certain limitations. First, nearly all (203/239, 85\%) patients were women. The reason for such predominance of Korean female patients remains unknown, although such predominance has been welldocumented $[38,39]$. Second, this study was a retrospective comparative study based on database of one institute. It was difficult to control confounding factors with possible selection bias. Therefore, it is difficult to generalize results of this study to other populations. We attempted to minimize population heterogeneity by excluding patients who used opioid and duloxetine prior to surgery. Third, because the same multimodal pain regimen including opioid was used until five days postoperatively during hospitalization, there was no result of early postoperative effect of pain control between the two groups. However, the purpose of this study was to see long-term pain control effects of 6-week administration of both drugs. Thus, we focused on efficacy of shortand long-term pain control in both groups. Finally, results of this study could only be extrapolated to patients undergoing TKA. Other orthopedic procedures might require further studies. Despite these limitations of the methodology, we believe that results of the present study have great strengths in that it is the first study about agents that could replace opioids in patients with persistent pain after TKA. 


\section{Conclusion}

In conclusion, duloxetine can provide comparable pain management to opioid with improved function for up to one year after TKA without increasing apparent risks of adverse effects. Our results indicate that duloxetine is a good alternative to opioid in a multimodal pain management protocol for patients who are discharged after TKA.

\section{Abbreviations}

TKA

Total Knee Arthroplasty

VAS

Visual Analogue Scale

WOMAC

Western Ontario and McMaster Universities OA Index

MCID

minimum clinically significant difference

SNRI

Selective serotonin and norepinephrine reuptake inhibitor

CNS

central nervous system

PS

posterior-stabilized

PCA

patient-controlled analgesia

\section{Declarations}

\section{Acknowledgements}

None.

\section{Funding}

Source of Funding, there was no external funding source in this investigation

\section{Availability of data and materials}

The datasets used and analyzed during the current study available are from the corresponding author upon reasonable request.

\section{Authors' contributions}

MSK collected the data, performed the measurement and analysis, participated in the study design and drafted the manuscript. IJK, KYC participated in the study design, supervised the analysis and helped to draft the manuscript. SCY collected the data, performed the measurement. YI designed the study, supervised the whole 
study process and helped to draft and review the manuscript. All authors read and approved the final manuscript.

\section{Ethical approval}

All procedures performed in studies involving human participants were in accordance with the ethical standards of the institutional and/or national research committee and with the 1964 Declaration of Helsinki and its later amendments or comparable ethical standards. This study has been approved by the Institutional Review Board (IRB) of Seoul St. Mary's Hospital and informed written consent for participation in the study was obtained.

\section{Consent for publication}

Consent for publication in the study was obtained.

\section{Competing interests}

The authors declare that they have no competing interests

\section{References}

1. Chan EY, Blyth FM, Nairn L, Fransen M. Acute postoperative pain following hospital discharge after total knee arthroplasty. Osteoarthritis Cartilage. 2013;21:1257-63.

2. Kehlet H, Dahl JB. The value of "multimodal" or "balanced analgesia" in postoperative pain treatment. Anesth Analg. 1993;77:1048-56.

3. Schwenk ES, Pozek JJ, Viscusi ER. Managing Prolonged Pain After Surgery: Examining the Role of Opioids. J Arthroplasty. 2018;33:3389-93.

4. Manchikanti L, Helm S 2nd, Fellows B, Janata JW, Pampati V, Grider JS, et al. Opioid epidemic in the United States. Pain Physician. 2012;15:Es9-38.

5. Eriksen J, Sjogren P, Bruera E, Ekholm O, Rasmussen NK. Critical issues on opioids in chronic non-cancer pain: an epidemiological study. Pain. 2006;125:172-9.

6. Furlan AD, Sandoval JA, Mailis-Gagnon A, Tunks E. Opioids for chronic noncancer pain: a meta-analysis of effectiveness and side effects. Cmaj. 2006;174:1589-94.

7. Hah JM, Sharifzadeh Y, Wang BM, Gillespie MJ, Goodman SB, Mackey SC, et al. Factors Associated with Opioid Use in a Cohort of Patients Presenting for Surgery. Pain Res Treat. 2015;2015:829696.

8. Chappell AS, Ossanna MJ, Liu-Seifert H, lyengar S, Skljarevski V, Li LC, et al. Duloxetine, a centrally acting analgesic, in the treatment of patients with osteoarthritis knee pain: a 13-week, randomized, placebocontrolled trial. Pain. 2009;146:253-60.

9. Pettersen VL, Zapata-Sudo G, Raimundo JM, Trachez MM, Sudo RT. The synergistic interaction between morphine and maprotiline after intrathecal injection in rats. Anesth Analg. 2009;109:1312-7.

10. Woolf CJ. Pain: moving from symptom control toward mechanism-specific pharmacologic management. Ann Intern Med. 2004;140:441-51.

11. Castro-Alves LJ, Oliveira de Medeiros AC, Neves SP, Carneiro de Albuquerque CL, Modolo NS, De Azevedo VL, et al. Perioperative Duloxetine to Improve Postoperative Recovery After Abdominal Hysterectomy: A 
Prospective, Randomized, Double-Blinded, Placebo-Controlled Study. Anesth Analg. 2016;122:98-104.

12. Sun YH, Dong YL, Wang YT, Zhao GL, Lu GJ, Yang J, et al. Synergistic analgesia of duloxetine and celecoxib in the mouse formalin test: a combination analysis. PLoS One. 2013;8:e76603.

13. Perahia DG, Kajdasz DK, Desaiah D, Haddad PM. Symptoms following abrupt discontinuation of duloxetine treatment in patients with major depressive disorder. J Affect Disord. 2005;89:207-12.

14. Charkhpour M, Jafari RM, Ghavimi H, Ghanbarzadeh S, Parvizpur A. Duloxetine attenuated morphine withdrawal syndrome in the rat. Drug Res (Stuttg). 2014;64:393-8.

15. Jones CK, Peters SC, Shannon HE. Efficacy of duloxetine, a potent and balanced serotonergic and noradrenergic reuptake inhibitor, in inflammatory and acute pain models in rodents. J Pharmacol Exp Ther. 2005;312:726-32.

16. Ho KY, Tay W, Yeo MC, Liu H, Yeo SJ, Chia SL, et al. Duloxetine reduces morphine requirements after knee replacement surgery. Br J Anaesth. 2010;105:371-6.

17. YaDeau JT, Brummett CM, Mayman DJ, Lin Y, Goytizolo EA, Padgett DE, et al. Duloxetine and Subacute Pain after Knee Arthroplasty when Added to a Multimodal Analgesic Regimen: A Randomized, Placebocontrolled, Triple-blinded Trial. Anesthesiology. 2016;125:561-72.

18. Lazzari M, Greco MT, Marcassa C, Finocchi S, Caldarulo C, Corli O. Efficacy and tolerability of oral oxycodone and oxycodone/naloxone combination in opioid-naive cancer patients: a propensity analysis. Drug Des Devel Ther. 2015;9:5863-72.

19. van der Wees PJ, Wammes JJ, Akkermans RP, Koetsenruijter J, Westert GP, van Kampen A, et al. Patientreported health outcomes after total hip and knee surgery in a Dutch University Hospital Setting: results of twenty years clinical registry. BMC Musculoskelet Disord. 2017;18:97.

20. Chappell AS, Desaiah D, Liu-Seifert H, Zhang S, Skljarevski V, Belenkov Y, et al. A double-blind, randomized, placebo-controlled study of the efficacy and safety of duloxetine for the treatment of chronic pain due to osteoarthritis of the knee. Pain Pract. 2011;11:33-41.

21. Andersen LO, Gaarn-Larsen L, Kristensen BB, Husted H, Otte KS, Kehlet H. Subacute pain and function after fast-track hip and knee arthroplasty. Anaesthesia. 2009;64:508-13.

22. Fischer HB, Simanski CJ, Sharp C, Bonnet F, Camu F, Neugebauer EA, et al. A procedure-specific systematic review and consensus recommendations for postoperative analgesia following total knee arthroplasty. Anaesthesia. 2008;63:1105-23.

23. Bedard NA, Pugely AJ, Westermann RW, Duchman KR, Glass NA, Callaghan JJ. Opioid Use After Total Knee Arthroplasty: Trends and Risk Factors for Prolonged Use. J Arthroplasty. 2017;32:2390-4.

24. Buvanendran A, Kroin JS, Tuman KJ, Lubenow TR, Elmofty D, Moric M, et al. Effects of perioperative administration of a selective cyclooxygenase 2 inhibitor on pain management and recovery of function after knee replacement: a randomized controlled trial. Jama. 2003;290:2411-8.

25. Kim KY, Anoushiravani AA, Chen KK, Roof M, Long WJ, Schwarzkopf R. Preoperative Chronic Opioid Users in Total Knee Arthroplasty-Which Patients Persistently Abuse Opiates Following Surgery? J Arthroplasty. 2018;33:107-12.

26. Boudreau D, Von Korff M, Rutter CM, Saunders K, Ray GT, Sullivan MD, et al. Trends in long-term opioid therapy for chronic non-cancer pain. Pharmacoepidemiol Drug Saf. 2009;18:1166-75.

27. Wallis L. 21st Century Cures Act-A Cure-All for Patients? Am J Nurs. 2016;116:19-20. 
28. Hansen CA, Inacio MCS, Pratt NL, Roughead EE, Graves SE. Chronic Use of Opioids Before and After Total Knee Arthroplasty: A Retrospective Cohort Study. J Arthroplasty. 2017;32:811-7.e1.

29. Wong K, Phelan R, Kalso E, Galvin I, Goldstein D, Raja S, et al. Antidepressant drugs for prevention of acute and chronic postsurgical pain: early evidence and recommended future directions. Anesthesiology. 2014;121:591-608.

30. Koh IJ, Kim MS, Sohn S, Song KY, Choi NY, In Y. Duloxetine Reduces Pain and Improves Quality of Recovery Following Total Knee Arthroplasty in Centrally Sensitized Patients: A Prospective, Randomized Controlled Study. J Bone Joint Surg Am. 2019;101:64-73.

31. Gilron I, Tu D, Holden RR, Jackson AC, DuMerton-Shore D. Combination of morphine with nortriptyline for neuropathic pain. Pain. 2015;156:1440-8.

32. Khoromi S, Cui L, Nackers L, Max MB. Morphine, nortriptyline and their combination vs. placebo in patients with chronic lumbar root pain. Pain. 2007;130:66-75.

33. Guerriero F, Sgarlata C, Marcassa C, Ricevuti G, Rollone M. Efficacy and tolerability of low-dose oral prolonged-release oxycodone/naloxone for chronic nononcological pain in older patients. Clin Interv Aging. 2015;10:1-11.

34. Recla JM. New and emerging therapeutic agents for the treatment of fibromyalgia: an update. J Pain Res. 2010;3:89-103.

35. Frakes EP, Risser RC, Ball TD, Hochberg MC, Wohlreich MM. Duloxetine added to oral nonsteroidal antiinflammatory drugs for treatment of knee pain due to osteoarthritis: results of a randomized, double-blind, placebo-controlled trial. Curr Med Res Opin. 2011;27:2361-72.

36. Wang ZY, Shi SY, Li SJ, Chen F, Chen H, Lin HZ, et al. Efficacy and Safety of Duloxetine on Osteoarthritis Knee Pain: A Meta-Analysis of Randomized Controlled Trials. Pain Med. 2015;16:1373-85.

37. Busse JW, Wang L, Kamaleldin M, Craigie S, Riva JJ, Montoya L, et al. Opioids for Chronic Noncancer Pain: A Systematic Review and Meta-analysis. Jama. 2018;320:2448-60.

38. Kim MS, Koh IJ, Jang SW, Jeon NH, In Y. Two- to Four-Year Follow-up Results of Total Knee Arthroplasty Using a New High-Flexion Prosthesis. Knee Surg Relat Res. 2016;28:39-45.

39. Koh IJ, Kim TK, Chang CB, Cho HJ. In Y. Trends in use of total knee arthroplasty in Korea from 2001 to 2010. Clin Orthop Relat Res. 2013;471:1441-50.

\section{Figures}




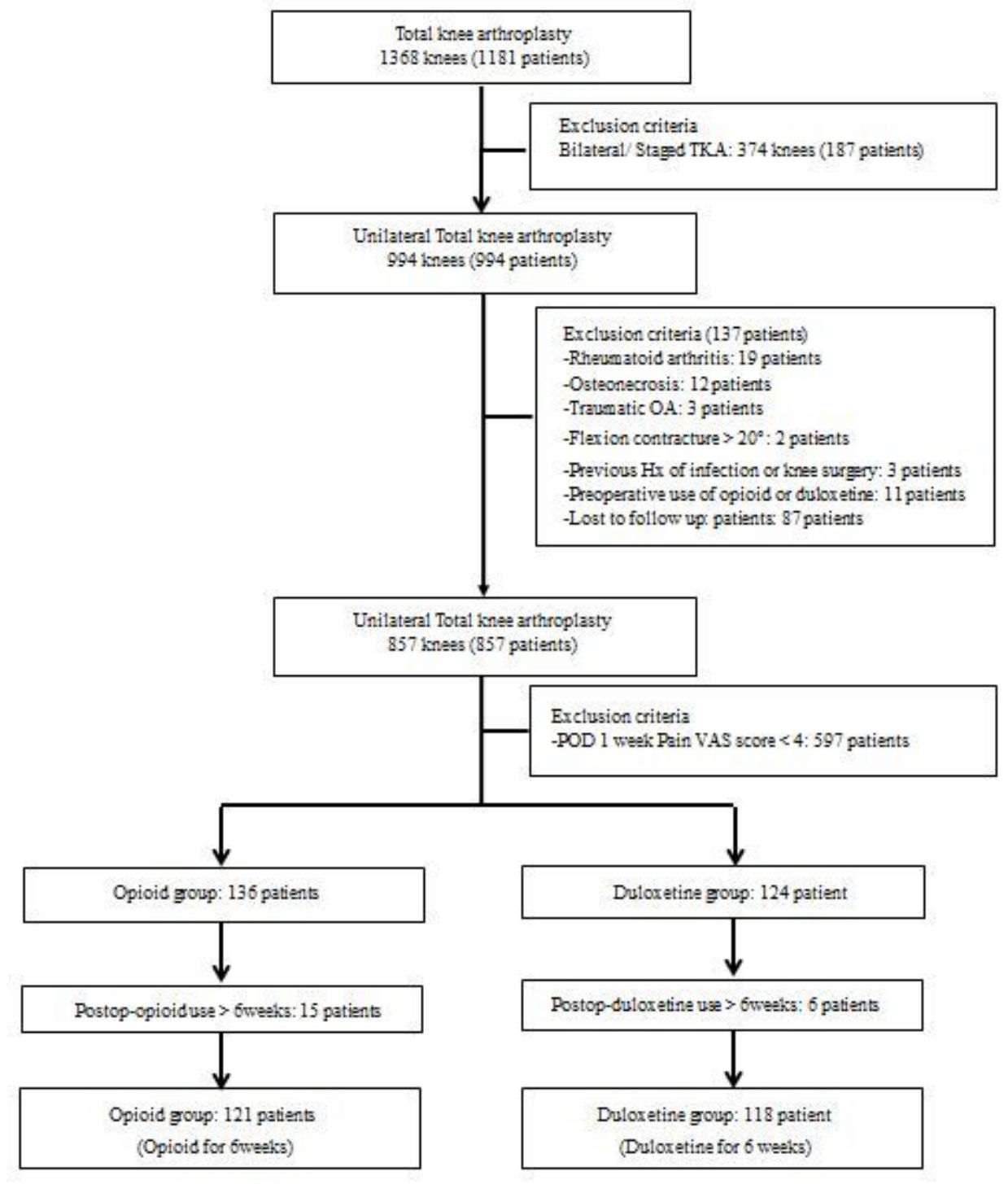

Figure 1

Flow chart describing patients invited to participate in this study and included in the final analysis 


\section{Range of Motion}

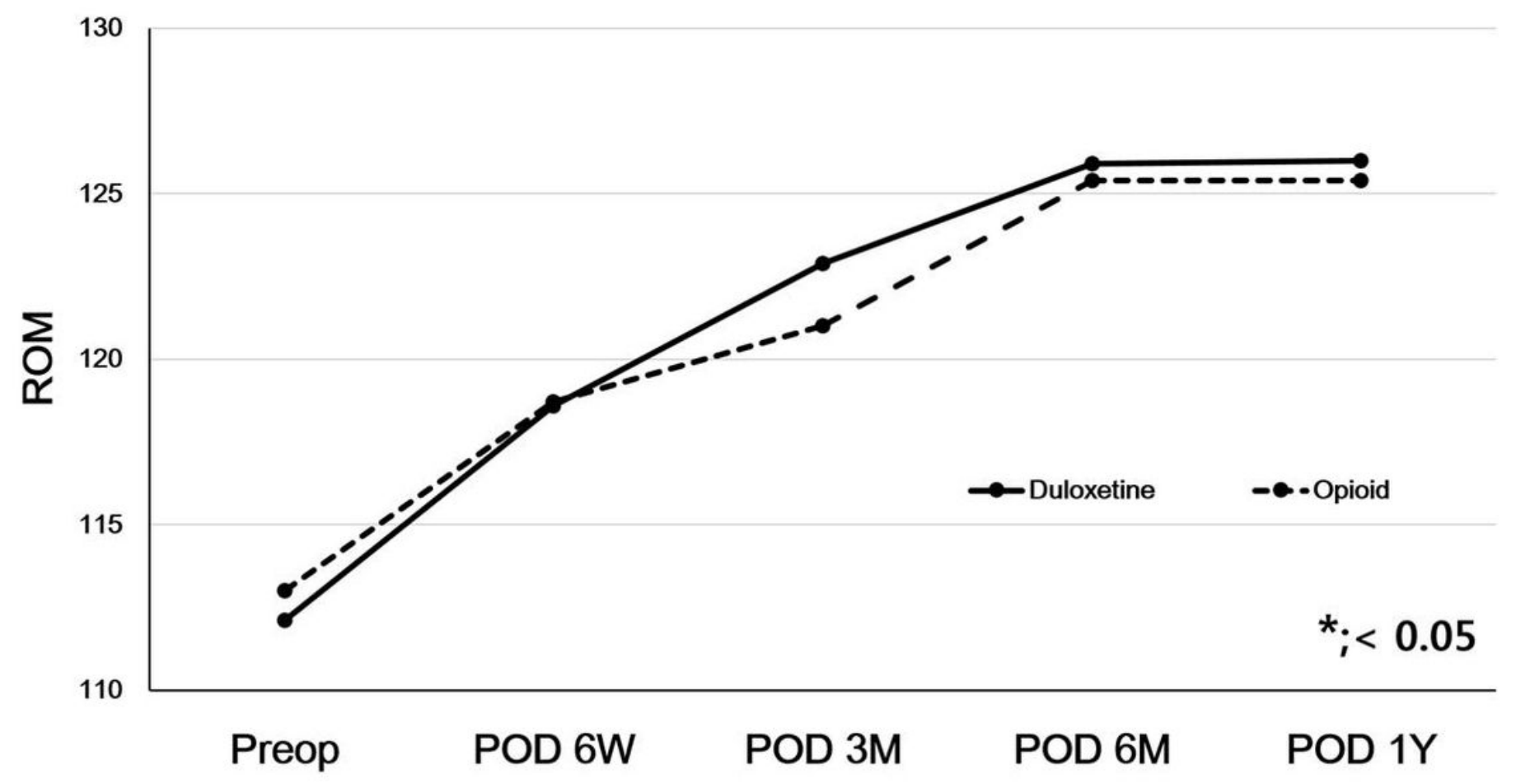

Figure 2

Comparisons of Range of Motion (ROM) between duloxetine and opioid groups. ROM did not differ between the two groups during postoperative period 

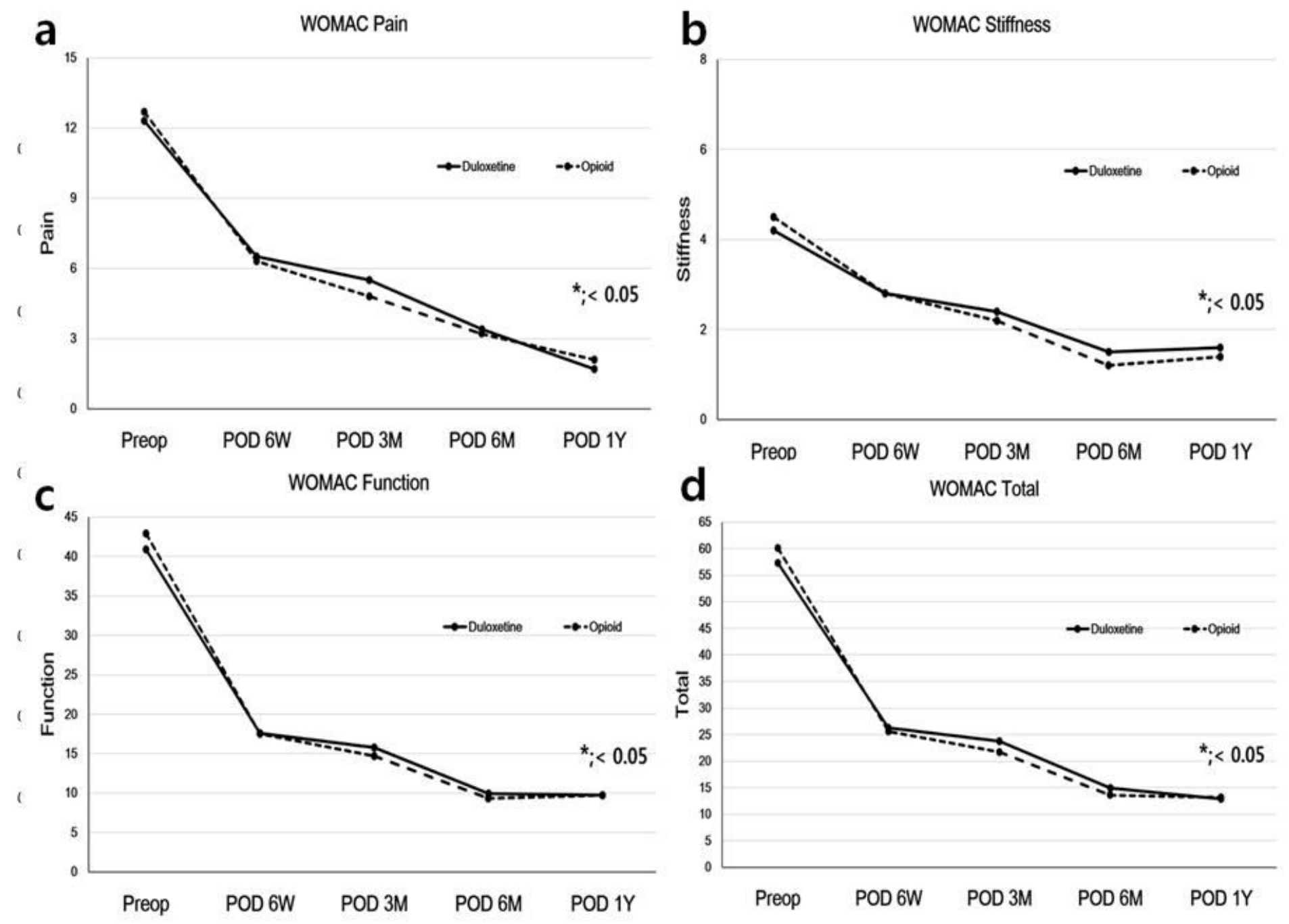

Figure 3

Comparisons of Western Ontario and McMaster Universities OA Index (WOMAC) subscores between duloxetine and opioid groups. There was no difference between the two groups regarding WOMAC pain (A), WOMAC stiffness (B), WOMAC function (C), or WOMAC total (D) at postoperative 6 weeks, 3 months, 6 months, or 1 year 